\title{
Initial combination anti-viral therapy with lamivudine and adefovir dipivoxil decreases short-term fatality rate of hepatitis-B-virus-related acute-on-chronic liver failure
}

\author{
Jiahong Yang ${ }^{*}$, Gao Chen, Xuebing Chen, Hao Zhang, Di Jiang and Guang Yang
}

\begin{abstract}
Background: Acute-on-chronic liver failure (ACLF) is a common serious hepatitis B virus (HBV)-related disease and has a poor prognosis. Until recently, initial combination antiviral treatment in ACLF patients was rarely reported. This study evaluated the effect of initial combination treatment with lamivudine and adefovir dipivoxil on the prognosis of HBV-related ACLF.

Methods: In this retrospective study, 131 eligible ACLF patients, including 61 treated with $100 \mathrm{mg}$ lamivudine and $10 \mathrm{mg}$ adefovir dipivoxil daily and 70 not treated with any nucleoside analogs (NAs), were selected and assigned into the NA and non-NA groups. All the patients received standard medicinal therapy. At weeks $0-4$ and 12 , serum markers for hepatic and renal functions were measured in all patients and accumulated fatality rates were calculated. Statistical analyses, including Student's $t$ test, $x^{2}$ test and unconditional logistic regression analysis, were performed using SPSS version 17.0 software.

Results: Clinical data indicated that improvement of hepatic function was better in the NA than in the non-NA group. The accumulated fatality rate in the NA group was lower than in the non-NA group at weeks 2-4 and 12, and these differences were significant. Univariate analysis showed that age, prothrombin activity, model of end-stage liver disease (MELD) score, and treatment without NAs were risk factors for short-term survival of ACLF. Further research by unconditional logistic regression analysis identified that older age, high MELD score and treatment without NAs were independent risk factors for short-term survival of ACLF.
\end{abstract}

Conclusions: Initial combination antiviral treatment is effective in decreasing short-term fatality of HBV-related ACLF.

Keywords: Hepatitis B virus, Acute-on-chronic liver failure, Antiviral treatment, Lamivudine, Adefovir dipivoxil

\section{Introduction}

Chronic hepatitis B virus (HBV) infection is a major public health problem worldwide. According to estimation of the World Health Organization, about 400 million people are chronically infected with $\mathrm{HBV}$ and $\sim 1$ million die from HBV-related diseases each year [1, 2]. Chronic HBV infection leads to several related liver diseases, including chronic hepatitis B, cirrhosis, hepatocellular carcinoma (HCC), and liver failure [3]. Acute-on-chronic liver failure (ACLF), defined in 1995 as a condition in which acute and chronic

\footnotetext{
* Correspondence: 18990283956@163.com

Department of Infectious Diseases, People's Hospital of Deyang City, No. 173, Taishan North Road, Deyang 618000, Sichuan Province, China
}

liver disease occur simultaneously, is a common serious HBV-related liver disease and causes acute deterioration of liver function [4]. ACLF has poor prognosis [5, 6].

There is no standard treatment for ACLF. The curative effect of standard medical treatment is limited and the fatality rate is high [7-9]. Clinical application of an artificial liver support system can temporarily improve patients' condition. However, it is not effective in decreasing fatality rate [10-13]. Although liver transplantation is effective for ACLF, its clinical application is hindered by the difficulty in finding suitable donors and its high cost $[14,15]$. Thus, a new treatment to improve prognosis is eagerly required in clinical practice.

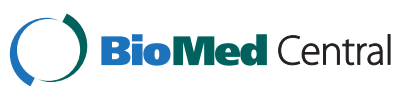


Although the mechanism of HBV-related ACLF remains obscure, growing evidence suggests that viral factors [16-18], host factors [19-21], and their interactions determine the outcome of ACLF. HBV DNA replication is an important factor for deterioration of liver function and closely associated with the risk of HCC.

Nucleoside analogues (NAs) can inhibit the replication of HBV and decrease serum HBV DNA loads, leading to improvement of liver function and reduction of cirrhotic complications and occurrence of HCC [22-24]. Treatment with one NA alone is unsatisfactory, although monotherapy can improve patients' condition and decrease short-term and long-term fatality rates [7-9]. Previous research has suggested that initial combination antiviral therapy with lamivudine and adefovir dipivoxil is more effective than monotherapy in decreasing serum HBV load and improving prognosis $[25,26]$. Until recently, initial combination antiviral therapy in ACLF patients was rarely reported. ACLF develops rapidly and has poor prognosis, and combination antiviral therapy is more effective than monotherapy $[25,26]$. Therefore, in the present study, 61 patients were treated with $100 \mathrm{mg}$ lamivudine and $10 \mathrm{mg}$ adefovir dipivoxil daily and 70 were not treated with any NA. Patients were selected to assess the effect of initial combination antiviral therapy on clinical condition and short-term survival of ACLF.

\section{Results}

\section{Baseline characteristics}

As mentioned above, 131 eligible ACLF patients were recruited and divided into NA and non-NA groups. There were no significant differences in the baseline characteristics of age, sex, hepatitis B e antigen ( $\mathrm{HBeAg}$ ) status, model of end-stage liver disease (MELD) score, and baseline levels of serum HBV DNA load, total bilirubin (TBIL), albumin, alanine aminotransferase (ALT), international normalized ratio (INR), prothrombin activity (PTA) and creatinine between the two groups (Table 1).

\section{Serum TBIL, ALT, albumin and PTA at different times of therapy}

Baseline serum TBIL, ALT, albumin and PTA did not differ significantly between the NA and non-NA groups. Serum TBIL levels increased at week 1, decreased below baseline levels at week 2, and then continued to decrease in the NA group (Table 2). In the non-NA group, serum TBIL levels continued to increase, reached a peak at week 2, and then began to decrease. Serum TBIL levels were lower in the NA group than non-NA group at weeks $1-4$ and these differences were significant at weeks 2 and $3(t=4.52, P<0.001 ; t=3.59, P=0.001)$ (Table 2). In the NA group, serum albumin levels continued to increase. In the non-NA group, serum albumin levels decreased below baseline levels at week 1 and then fluctuated at weeks 2-4. Serum albumin levels were higher in the NA group than non-NA group at weeks 1-4 and theses differences were significant $(t=3.67, P<0.001$; $t=3.92, P<0.001 ; t=5.77, P<0.001 ; t=3.88, P<0.001)$ (Table 2). Although serum PTA levels continued to increase in the NA and non-NA groups, it increased more rapidly in the NA group, and serum PTA levels were higher in the NA than non-NA group at weeks $1-4$. These differences were significant at weeks 2 and $3(t=2.52$, $P=0.013 ; t=2.38, P=0.020)$. Serum ALT levels continued to decrease in these two groups although the differences between the groups were not significant. There was no patient with abnormal serum creatinine and urea nitrogen levels in either group until week 12.

\section{Accumulated fatality rates in the two groups}

All ACLF patients in these two groups were followed up to week 12. At week 12, 55 patients were dead and the fatality rate was $41.98 \%$. Accumulated fatality rate at weeks $2-4$ and 12 in the NA group was $1.64 \%(1 / 61)$, $14.75 \%$ (9/61), $21.31 \%(13 / 61)$ and $24.59 \%$ (15/61), respectively. In the non-NA group, accumulated fatality rate was $21.43 \%$ (15/70), $35.71 \%$ (25/70), $47.14 \%$ (33/70) and

Table 1 Baseline characteristics of the patients

\begin{tabular}{|c|c|c|c|c|}
\hline & NA group (mean $\pm S D$ ) & Non-NA group (mean \pm SD) & tor $x^{2}$ value & $P$ value \\
\hline Male (\%) & $52(85.25)$ & $59(84.29)$ & 0.02 & 0.88 \\
\hline HBeAg positive (\%) & $19(31.15)$ & $18(25.71)$ & 0.48 & 0.49 \\
\hline Age (yr) & $40.31 \pm 10.75$ & $43.14 \pm 10.37$ & -1.53 & 0.13 \\
\hline HBV DNA $\log _{10}($ copies/ml) & $5.72 \pm 1.38$ & $5.31 \pm 1.56$ & 1.53 & 0.13 \\
\hline TBIL $(\mu \mathrm{mol} / \mathrm{L})$ & $278.56 \pm 116.09$ & $260.24 \pm 115.50$ & 0.90 & 0.37 \\
\hline Albumin $(\mathrm{g} / \mathrm{L})$ & $34.73 \pm 5.85$ & $34.35 \pm 4.49$ & 0.42 & 0.67 \\
\hline $\mathrm{ALT}(\mathrm{U} / \mathrm{L})$ & $1037.44 \pm 823.50$ & $833.93 \pm 794.70$ & 1.44 & 0.15 \\
\hline PTA (\%) & $31.15 \pm 9.71$ & $33.34 \pm 10.20$ & -1.25 & 0.21 \\
\hline Creatinine $(\mu \mathrm{mol} / \mathrm{L})$ & $66.01 \pm 12.46$ & $76.94 \pm 52.23$ & -1.70 & 0.09 \\
\hline MELD Score & $22.82 \pm 2.99$ & $23.95 \pm 5.91$ & -1.40 & 0.17 \\
\hline
\end{tabular}


Table 2 Time-dependent changes of serum TBIL, ALT, albumin and PTA

\begin{tabular}{|c|c|c|c|c|c|}
\hline Serum marker & Time & NA group & Non-NA group & $t$ value & $P$ value \\
\hline \multirow[t]{4}{*}{ TBIL ( $\mu \mathrm{mol} / \mathrm{L})$} & Week 1 & $310.98 \pm 134.94$ & $360.39 \pm 122.11$ & 1.95 & 0.054 \\
\hline & Week 2 & $272.74 \pm 166.83$ & $427.22 \pm 189.74$ & 4.52 & $<0.001$ \\
\hline & Week 3 & $239.22 \pm 172.03$ & $379.72 \pm 200.63$ & 3.59 & 0.001 \\
\hline & Week 4 & $240.55 \pm 210.19$ & $299.47 \pm 195.52$ & 1.10 & 0.28 \\
\hline \multirow[t]{4}{*}{$\mathrm{ALT}(\mathrm{U} / \mathrm{L})$} & Week 1 & $224.43 \pm 234.97$ & $267.75 \pm 242.43$ & -0.93 & 0.36 \\
\hline & Week 2 & $120.20 \pm 116.55$ & $122.05 \pm 89.96$ & -0.09 & 0.93 \\
\hline & Week 3 & $87.31 \pm 74.70$ & $73.96 \pm 38.98$ & 1.02 & 0.31 \\
\hline & Week 4 & $63.62 \pm 49.75$ & $57.48 \pm 28.60$ & 0.56 & 0.58 \\
\hline \multirow[t]{4}{*}{ Albumin ( $g / L)$} & Week 1 & $35.31 \pm 5.09$ & $32.05 \pm 3.74$ & 3.67 & $<0.001$ \\
\hline & Week 2 & $36.78 \pm 5.02$ & $33.37 \pm 3.78$ & 3.92 & $<0.001$ \\
\hline & Week 3 & $37.51 \pm 5.41$ & $32.39 \pm 2.90$ & 5.77 & $<0.001$ \\
\hline & Week 4 & $37.38 \pm 5.21$ & $32.42 \pm 4.41$ & 3.88 & $<0.001$ \\
\hline \multirow[t]{4}{*}{ PTA (\%) } & Week 1 & $52.77 \pm 27.31$ & $45.59 \pm 24.93$ & 1.34 & 0.18 \\
\hline & Week 2 & $57.85 \pm 29.73$ & $44.31 \pm 23.20$ & 2.52 & 0.013 \\
\hline & Week 3 & $65.58 \pm 30.56$ & $49.70 \pm 27.53$ & 2.38 & 0.020 \\
\hline & Week 4 & $58.23 \pm 31.56$ & $48.50 \pm 24.78$ & 1.14 & 0.260 \\
\hline
\end{tabular}

$57.14 \%(40 / 70)$ at weeks $2-4$ and 12 , respectively. The accumulated fatality rates in the NA group were lower than in the non-NA group and these differences were significant $\left(X^{2}=11.91, P=0.001 ; X^{2}=7.45, P=0.006 ; X^{2}=9.55\right.$, $\left.P=0.002 ; X^{2}=14.18, P<0.001\right)$. This indicated that antiviral therapy may decrease short-term fatality rate.

\section{Factors influencing short-term survival of ACLF}

Univariate analysis was performed to identify risk factors for short-term survival of ACLF patients. Age, PTA, MELD score and treatment without NAs were risk factors for short-term survival of ACLF patients $(t=3.06$, $P=0.003 ; t=2.05, P=0.042 ; t=3.49, P=0.001 ; \chi^{2}=14.18$, $P<0.001$ ) (Table 3 ). Multivariate analysis using unconditional logistic regression was performed to identify independent risk factors for short-term survival of ACLF patients and $P \geq 0.05$ was selected as the exclusion criterion. Sex, age, HBeAg status, HBV DNA loads, MELD score, serum TBIL level, ALT level, albumin level and PTA level, and treatment without NAs were included in the unconditional regression analysis. Unconditional logistic regression analysis showed that age, MELD score and treatment without NAs were independent risk factors for short-term survival of ACLF $[P=0.007$, odds ratio $(\mathrm{OR})=1.063$, $95 \%$ confidence interval $(\mathrm{CI}): 1.017-1.112$; $P=0.002$, OR $=1.201,95 \%$ CI: $1.068-1.351 ; P=0.001$, $\mathrm{OR}=4.717,95$ \% CI: 1.967-11.315) (Table 4).

\section{Discussion}

ACLF is a serious liver disease caused by multiple factors, with impairment of several major liver functions, including synthetic function, detoxification and metabolic regulation.

Table 3 Univariate analysis of risk factors for the survival of ACLF patients

\begin{tabular}{|c|c|c|c|c|}
\hline Related factors & Survivor $(n=76)($ Mean \pm SD $)$ & Non-survivor $(n=55)($ Mean \pm SD) & $t$ or $x^{2}$ value & $P$ value \\
\hline Male (\%) & $64(84.21)$ & $47(85.45)$ & 0.04 & 0.85 \\
\hline HBeAg positive (\%) & $19(25.00)$ & $18(32.73)$ & 0.94 & 0.33 \\
\hline Treatment without NAs (\%) & $30(39.47)$ & $40(72.73)$ & 14.18 & $<0.001$ \\
\hline Age (yr) & $39.49 \pm 10.50$ & $45.05 \pm 9.96$ & 3.06 & 0.003 \\
\hline HBV DNA $\log _{10}($ copies/ml) & $5.52 \pm 1.40$ & $5.50 \pm 1.60$ & 0.08 & 0.94 \\
\hline TBIL ( $\mu \mathrm{mol} / \mathrm{L})$ & $271.33 \pm 111.05$ & $265.23 \pm 122.78$ & 0.30 & 0.77 \\
\hline Albumin (g/L) & $34.67 \pm 5.44$ & $34.34 \pm 4.77$ & 0.37 & 0.71 \\
\hline $\operatorname{ALT}(U / L)$ & $912.92 \pm 861.61$ & $950.50 \pm 744.03$ & 0.26 & 0.80 \\
\hline PTA (\%) & $33.81 \pm 9.40$ & $30.21 \pm 10.50$ & 2.05 & 0.042 \\
\hline Creatinine $(\mu \mathrm{mol} / \mathrm{L})$ & $66.66 \pm 14.74$ & $79.03 \pm 57.78$ & 1.55 & 0.13 \\
\hline MELD Score & $22.11 \pm 2.84$ & $25.24 \pm 6.19$ & 3.49 & 0.001 \\
\hline
\end{tabular}


Table 4 Multivariate analysis of risk factors for the survival of ACLF patients

\begin{tabular}{lllllll}
\hline Related factors & Regression coefficient (B) & Standard error & Wals & $P$ & OR value & $95 \% \mathrm{Cl}$ \\
\hline Age $(\mathrm{yr})$ & 0.061 & 0.023 & 7.355 & 0.007 & 1.063 & $1.017-1.112$ \\
Treatment without NAs & 1.551 & 0.446 & 12.077 & 0.001 & 4.717 & $1.967-11.315$ \\
HBV DNA $\log _{10}$ (copies/ml) & 0.310 & 0.164 & 3.584 & 0.058 & 1.363 & $0.989-1.879$ \\
MELD score & 0.183 & 0.060 & 9.356 & 0.002 & 1.201 & $1.068-1.351$ \\
Cons & -9.717 & 2.306 & 17.761 & $<0.001$ & - \\
\hline
\end{tabular}

In addition, it can lead to ascites, jaundice, cholestasis, bleeding, hepatic encephalopathy, and hepatorenal syndrome [5]. Fatality rate of ACLF is high and the prognosis of ACLF is poor [6].

Previous research has revealed that $\mathrm{HBV}$ replication and high serum HBV load are key factors for severe impairment of liver function and associated with rapid disease progression of ACLF. Previously research revealed that antiviral treatment of ACLF could effectively decrease fatality rate and improve patients' quality of life [27]. A study from Taiwan suggested that antiviral therapy increases short-term survival rate of ACLF at the early stage of liver failure, although it is not able to increases short-term survival rate of ACLF at the late stage of liver failure. Antiviral treatment as early as possible is more effective in represses viral replication, preventing deterioration of clinical condition and improving prognosis of ACLF. Lamivudine has been proven effective in inhibiting viral replication, improving biochemical and histology, and reducing the inflammatory response. Lamivudine treatment has been shown useful for the patients with HBV-related ACLF [28]. Adefovir dipivoxil, a phosphonate nucleoside analog of AMP, represses polymerase activity of $\operatorname{HBV}[23,29]$, and suppresses replication of wild-type and lamivudine-resistant HBV. Previous research has suggested that combination antiviral treatment with lamivudine and adefovir dipivoxil is more effective at decreasing serum HBV load in chronic hepatitis B patients than using one nucleoside [25, 26]. ACLF usually develops rapidly, and patients are in poor condition and have a poor prognosis. Initial combination antiviral treatment with lamivudine and adefovir dipivoxil can improve prognosis of ACLF through repression $\mathrm{HBV}$ replication rapidly and effectively. Until recently, initial combination antiviral treatment in ACLF patients has rarely been reported. This prompted us to investigate the effect of initial combination antiviral treatment with lamivudine plus adefovir dipivoxil in ACLF patients.

In our study, the TBIL, ALB and PTA were significant improved at 1 week to 4 week in the NA group. While in the non-NA group, the TBIL, ALB and PTA were not effectively improved as the duration of treatment. Previous research showed that the 3-month accumulated fatality rate in patients treated with entecavir, lamivudine and
non-NAs was $51.51 \%, 50 \%$ and $59.46 \%$, respectively. The difference in 3-month accumulated fatality rate between patients treated with NAs or not was not significant [7]. Another research reported that the 3-month accumulated fatality rate in patients treated with entecavir, lamivudine and non-NAs was $30.95 \%, 40 \%$ and $64.71 \%$, respectively [8]. In this study, patients treated with NAs had a significantly lower accumulated fatality rate than patients treated without NAs. Our research suggested that the accumulated fatality rates in the NA group were lower than in the non-NA group at weeks $2-4$ and 12 , and that these differences were significant. The 12-week accumulated fatality rate was $24.59 \%$ and $57.14 \%$ in the NA and non-NA group, respectively. In our study,univariate analysis showed that age, MELD score, PTA and treatment without NAs were risk factors for short-term survival of ACLF patients. Multivariate analysis by unconditional logistic regression suggested that age, MELD score and treatment without NAs were independent risk factors for fatality rate at short-term in ACLF patients. Our results showed that initial combination antiviral treatment significantly decreased short-term accumulated fatality rate of ACLF patients. It is likely that initial combination antiviral treatment with lamivudine plus adefovir dipivoxil may be more effective in inhibiting $\mathrm{HBV}$ replication, decreasing serum HBV DNA loads, lessening inflammation impairment in hepatic tissue, and improving prognosis.

Our study has potential weakness. First, our research is a retrospective research during May 2006 to June 2011. At that time, lamivudine and adefovir dipivoxil are the most widely used nucleoside analogues in China. So, our study only investigated the initial antiviral treatment with lamivudine plus adefovir dipivoxil. In the future, we should investigate antiviral treatment effect of other nucleoside analogues such as tenofovir, entecavir and telbivudine in ACLF patients. Second, the sample size of our research is relatively small. Next step, we should verify our results in a large sample size.

\section{Conclusions}

Our results revealed that initial combination antiviral treatment with lamivudine plus adefovir dipivoxil is able to improve biochemical parameters, increase PTA and decreased short-term fatality rate of ACLF patients. 


\section{Methods}

\section{Patients}

ACLF was diagnosed according to "Diagnostic and Treatment Guidelines for Liver Failure (2012 version)" recommended by the Chinese Medical Association [4]. The inclusion criteria were as follows: (1) age 18-65 years; (2) hepatitis B surface antigen (HBsAg) positive for >6 months; (3) serum HBV DNA level >3 $\log _{10}$ copies/ml; (4) recent development of increasing jaundice (serum bilirubin concentration $>171 \mu \mathrm{mol} / \mathrm{l}$ ) and decreasing plasma PTA $(\leq 40 \%)$ or INR $>1.5$; and (5) recent development of complications such as hepatic encephalopathy, abrupt and obvious increase in ascites, spontaneous bacterial peritonitis, or hepatorenal syndrome.

Patients affected by other viral hepatitis, alcohol hepatitis, autoimmune hepatitis, drug-induced hepatitis, primary liver cancer and hepatorenal syndrome were excluded. Patients with jaundice caused by obstructive or hemolytic diseases or prolonged prothrombin time induced by blood system diseases were also excluded.

A total of 131 eligible HBV-related ACLF patients were included in our retrospective study. Seventy patients hospitalized in the Department of Infectious Diseases, People's Hospital of Deyang City from May 2006 to January 2009, were not treated with NAs and assigned to the non-NA group. Sixty-one patients hospitalized from February 2009 to June 2011 were treated with lamivudine plus adefovir and assigned to the NA group. No patients were previously treated with nucleoside analogues and interferon. Our study protocol conformed to the ethical guidelines of the 1975 Declaration of Helsinki and was approved by the Ethics Committee of People's Hospital of Deyang City.

\section{Treatment schedule}

All patients received standard medical treatment, including supply of energy, fresh plasma and albumin, maintenance of water, electrolyte and acid-base equilibrium, promoting growth of hepatic cells, anti-inflammatory and antioxidant treatments, and liver protection methods. Patients with spontaneous peritonitis received anti-infective therapy using third-generation cephalosporins. Besides standard internal medical therapy, patients in the NA group received initial combination antiviral treatment with lamivudine (100 mg daily) (GlaxoSmithKline, Greenford, UK) plus adefovir dipivoxil (10 mg daily) (GlaxoSmithKline) within $24 \mathrm{~h}$ after hospitalization.

\section{Measurement of serum markers}

Serum markers reflecting hepatocyte damage, including serum ALT, albumin, TBIL, and creatinine, were assayed by colorimetry (Hitachi 7180; Hitachi, Tokyo, Japan). Serum HBV markers, including HBsAg, hepatitis B surface antibody, $\mathrm{HBeAg}$, hepatitis $\mathrm{B}$ e antibody and hepatitis B core antibody, were detected by time-resolved fluorescence immunoassay (Tailai-II, Fenghua, Guangzhou, China). PTA was measured by solidification method and INR was calculated according to the manufacturer's instructions (Sysmex CA 7000; Sysmex, Kobe, Japan). Serum HBV DNA load was quantified by Quantitive Diagnostic Kit for Hepatitis B Virus DNA (Qiagen, Shenzhen, Guangdong, China). The MELD score was calculated using the equation: MELD score $=3.78 \times \ln \quad[$ total bilirubin $(\mathrm{mg} / \mathrm{dL})]+11.2 \times \ln$ $\mathrm{INR}+9.57 \times \ln [$ creatinine $(\mathrm{mg} / \mathrm{dL})]+6.43[30]$.

\section{Follow-up}

Patients were followed up at weeks $1-4$ and 12 . Clinical and laboratory data and adverse events were recorded.

\section{Statistical analyses}

Statistical analyses, including Student's $t$ test, $\chi^{2}$ test and unconditional logistic regression, were performed by SPSS version 17.0. $P<0.05$ was considered statistically significant.

\section{Abbreviations}

ACLF: Acute-on-chronic liver failure; ALT: Alanine aminotransferase;

HBeAg: Hepatitis B e antigen; HBsAg: Hepatitis B surface antigen; HBV: Hepatitis B virus; INR: International normalized ratio; MELD: Model for end-stage liver disease: NA: Nucleoside analog; PTA: Prothrombin activity; TBIL: Total bilirubin.

\section{Competing interests}

The authors declare that they have no competing interests.

\section{Authors' contributions}

$J Y$ designed the research and wrote the manuscript, GC and XC analyzed data and prepared the manuscript, $\mathrm{HZ}$ prepared the manuscript, DJ and GY involved in follow-up of these patients. All authors read and approved the final manuscript.

\section{Acknowledgments}

This work was supported by grants from Science and Technology Bureau of Deyang City, Sichuan Province, China (No. 2011SZ040-3).

Received: 3 July 2014 Accepted: 10 June 2015

Published online: 24 June 2015

\section{References}

1. Lavanchy D. Hepatitis B, virus epidemiology, disease burden, treatment, and current and emerging prevention and control measures. J Viral Hepatitis. 2004;11:97-107.

2. Alter M. Epidemiology of hepatitis B in Europe and worldwide. J Hepatol. 2003:39:564-9.

3. Lee WM. Hepatitis B, virus infection. N Engl J Med. 1997;337:1733-45

4. Li L. Diagnostic and treatment guidelines for liver failure (2012 version). Chin J Hepatol. 2013;21:177-83.

5. Jalan R, Williams R. Acute-on-chronic liver failure: pathophysiological basis of therapeutic options. Blood Purif. 2002;20:252-61.

6. Seto WK, Lai CL, Yuen MF. Acute-on-chronic liver failure in chronic hepatitis B. J Gastroenterol Hepatol. 2012;27:662-9.

7. Cui YL, Yan F, Wang YB, Song XQ, Liu L, Lei $X Z$, et al. Nucleoside analogue can improve the long-term prognosis of patients with hepatitis $B$ virus infection-associated acute on chronic liver failure. Dig Dis Sci. 2010;55:2373-80.

8. Chen T, He Y, Liu X, Yan Z, Wang K, Liu H, et al. Nucleoside analogues improve the short-term and long-term prognosis of patients with hepatitis B virus-related acute-on-chronic liver failure. Clin Exp Med. 2012;12:159-64.

9. Liu XY, Wang HF, Hu JH, He WP, Wang HQ, Liu N. The short-term efficacy of nucleoside analogue on the treatment of acute-on-chronic liver failure. Zhonghua Gan Zang Bing Za Zhi. 2010;18:845-8. 
10. Xu X, Liu X, Ling Q, Wei Q, Liu Z, Xu X, et al. Artificial liver support system combined with liver transplantation in the treatment of patients with acute-on-chronic liver failure. PLoS One. 2013;8:e58738.

11. Stadlbauer V, Davies NA, Sen S, Jalan R. Artificial liver support systems in the management of complications of cirrhosis. Semin Liver Dis. 2008;28:96-109.

12. Santoro A, Mancini E, Ferramosca E, Faenza S. Liver support systems. Contrib Nephrol. 2007;156:396-404.

13. Abe T, Kobata H, Hanba Y, Kitabata Y, Narukawa N, Hasegawa H, et al. Study of plasma exchange for liver failure: beneficial and harmful effects. Ther Apher Dial. 2004;8:180-4

14. Bahirwani R, Shaked O, Bewtra M, Forde K, Reddy KR. Acute-on-chronic liver failure before liver transplantation: impact on posttransplant outcomes. Transplantation. 2011;92:952-7.

15. Chan AC, Fan ST, Lo CM, Liu CL, Chan SC, Ng KK, et al. Liver transplantation for acute-on-chronic liver failure. Hepatol Int. 2009;3:571-81.

16. Yan T, Li K, Su HB, Liu XY, Zang H, Wang HF. Analysis the relationship of HBV BCP A1762T/G1764A double mutation with HBV related acute on chronic liver failure. Zhonghua Shi Yan He Lin Chuang Bing Du Xue Za Zhi. 2010;24:190-2

17. Xu Z, Ren X, Liu Y, Li X, Bai S, Zhong Y, et al. Association of hepatitis B virus mutations in basal core promoter and precore regions with severity of liver disease: an investigation of 793 Chinese patients with mild and severe chronic hepatitis B and acute-on-chronic liver failure. J Gastroenterol. 2011:46:391-400

18. Ren X, Xu Z, Liu Y, Li X, Bai S, Ding N, et al. Hepatitis B virus genotype and basal core promoter/precore mutations are associated with hepatitis B-related acute-on-chronic liver failure without pre-existing liver cirrhosis. J Viral Hepat. 2010;17:887-95.

19. Zhang JY, Zhang Z, Lin F, Zou ZS, Xu RN, Jin L, et al. Interleukin-17-producing CD4(+) T cells increase with severity of liver damage in patients with chronic hepatitis B. Hepatology. 2010;51:81-91.

20. Song $\mathrm{QL}, \mathrm{He} X X$, Yang H, Li J, Chen M, Wang MY, et al. Association of a TANK gene polymorphism with outcomes of hepatitis $B$ virus infection in a Chinese Han population. Viral Immunol. 2012;25:73-8.

21. Rong Y, Song H, You S, Zhu B, Zang H, Zhao Y, et al. Association of Toll-like receptor 3 polymorphisms with chronic hepatitis $B$ and hepatitis B-related acute-on-chronic. liver failure. Inflammation. 2013;36:413-8.

22. Hache C, Villeneuve JP. Lamivudine treatment in patients with chronic hepatitis B and cirrhosis. Expert Opin Pharmacother. 2006;7:1835-43.

23. Qaqish RB, Mattes KA, Ritchie DJ. Adefovir dipivoxil: a new antiviral agent for the treatment of hepatitis B virus infection. Clin Ther. 2003:25:3084-99.

24. Liaw YF, Sung JJ, Chow WC, Farrell G, Lee CZ, Yuen H, et al. Cirrhosis Asian Lamivudine Multicentre Study Group: Lamivudine for patients with chronic hepatitis B and advanced liver disease. N Engl J Med. 2004;351:1521-31.

25. Liu F, Wang $X$, Wei F, Hu H, Zhang D, Hu P, et al. Efficacy and resistance in de novo combination lamivudine and adefovir dipivoxil therapy versus entecavir monotherapy for the treatment-naive patients with chronic hepatitis B: a meta-analysis. Virol J. 2014;11:59.

26. Zhang J. De novo combination therapy with lamivudine and adefovir dipivoxil versus entecavir monotherapy for naive chronic hepatitis B patients with high viral load. Chin J Clin Infect Di. 2012;3:142-4.

27. Yu S, Jianqin H, Wei W, Jianrong H, Yida Y, Jifang S, et al. The efficacy and safety of nucleos(t)ide analogues in the treatment of HBV-related acute-on-chronic liver failure: a meta-analysis. Ann Hepatol. 2013;12(3):364-72.

28. Tan $Y$, Ding $K$, Su J, Trinh $X$, Peng Z, Gong Y, et al. The naturally occurring YMDD mutation among patients chronically infected HBV and untreated with lamivudine: a systematic review and meta-analysis. PLoS One. 2012;7: e32789.

29. Danta M, Dusheiko G. Adefovir dipivoxil: review of a novel acyclic nucleoside analogue. Int J Clin Pract. 2004;58:877-86.

30. Malinchoc M, Kamath PS, Gordon FD, Peine CJ, Rank J, ter Borg PC. A model to predict poor survival in patients undergoing transjugular intrahepatic portosystemic shunts. Hepatology. 2000;31:864-71.

\section{Submit your next manuscript to BioMed Central and take full advantage of:}

- Convenient online submission

- Thorough peer review

- No space constraints or color figure charges

- Immediate publication on acceptance

- Inclusion in PubMed, CAS, Scopus and Google Scholar

- Research which is freely available for redistribution

Submit your manuscript at www.biomedcentral.com/submit 